\title{
A Report of Cat Scratch Disease in Korea Confirmed by PCR Amplification of the 16S-23S rRNA Intergenic Region of Bartonella benselae
}

\author{
Borum Suh, M.D. ${ }^{1}$, Jin-Kyoung Chun, M.D. ${ }^{2}$, Dongeun Yong, M.D. ${ }^{1,4}$, Yang Soon Lee, M.D..$^{1,4}$, Seok Hoon Jeong, M.D. ${ }^{1,4}$, \\ Woo Ick Yang, M.D. ${ }^{3}$, and Dong Soo Kim, M.D. ${ }^{2}$ \\ Departments of Laboratory Medicine ${ }^{1}$, Pediatrics², and Pathology ${ }^{3}$, Research Institute of Bacterial Resistance ${ }^{4}$, \\ Yonsei University College of Medicine, Seoul, Korea
}

\begin{abstract}
We report a case of cat scratch disease in an 8-yr-old girl who presented with fever and enlargement of both axillary lymph nodes. Both aerobic and anaerobic cultures of the lymph node aspirate were negative for microbial growth. Gram staining and Warthin-Starry silver staining did not reveal any organism. Purified DNA from the PCR-amplicon of the 16S-23S rRNA intergenic region was sequenced and showed $99.7 \%$ identity with the corresponding sequence of Bartonella henselae strain Houston-1. Our findings suggest that the internal transcribed spacer is a reliable region for PCR identification of Bartonella species. In patients with lymphadenitis, a history of contact with cats or dogs necessitates the use of diagnostic approaches that employ not only the conventional staining and culture but also molecular methods to detect B. henselae. (Korean J Lab Med 2010;30:34-7)
\end{abstract}

Key Words : Bartonella henselae, Cat scratch disease, Polymerase chain reaction

Cat scratch disease (CSD) is a benign disease characterized by fever, self-limited regional lymphadenitis, and skin lesions due to cat scratch or bite. CSD shows a worldwide distribution and has been mostly reported in children aged less than 18 yr of age. The etiologic agent of CSD is Bartonella henselae, a gram-negative rod [1]. Little is known about the epidemiology of CSD in Korea. Since B. henselae is fastidious and cannot be grown in cultures, only 2 CSD cases have been reported using molecular genetic methods in Korea [2, 3]. Here, we present the third case of CSD in a Korean pediatric patient; the disease was confirmed using PCR and DNA sequencing with primers recently described in the literature.

In September 2008, an 8-yr-old girl presented with

Received: September 26, 2009

Manuscript No : KJLM09-118

Revision received : November 23, 2009

Accepted: December 9, 2009

Corresponding author: Dong Eun Yong, M.D.

Department of Laboratory Medicine, Yonsei University College of Medicine, 250 Seongsan-ro, Seodaemun-gu, Seoul 120-752, Korea

Tel : +82-2-2228-2445 Fax : +82-2-313-0956

E-mail : deyong@yuhs.ac enlargement of both axillary lymph nodes and fever that had persisted for 2 weeks. The patient had received all the recommended immunizations. A month before her admission, a cat had scratched her on the right subclavicular area. Investigation of her family history revealed that her grandmother had been diagnosed with pulmonary tuberculosis.

Physical examination of the patient revealed a $2 \times 2 \mathrm{~cm}$ mass on the right axillary lymph node and a $3.5 \times 3 \mathrm{~cm}$ tender and slightly movable mass on the left axillary lymph node. The skin on the right chest wall showed papular lesions. The liver, spleen, or other lymph nodes did not show any apparent enlargement. Yellowish pus was exuded from the left axillary lymph node.

The initial complete blood cell count showed that the WBC count was $14,710 / \mu \mathrm{L}$; platelet count, $445,000 / \mu \mathrm{L}$; and hemoglobin level, $11.1 \mathrm{~g} / \mathrm{dL}$. Mycobacterium tuberculosis antigen-specific interferon-gamma release assay of her serum sample by using QuantiFERON ${ }^{\circledR}$-TB Gold IT (Cellestis, Ltd., Carnegie, Australia) and PCR of the 
lymph node aspirate by using AMPLICOR Mycobacterium kit ${ }^{\mathrm{TM}}$ (Roche Diagnostic Systems, Inc., Branchburg, NJ, USA) yielded negative results.

Gram staining of the pus aspirate did not reveal any organism. Air-dried slides of the pus aspirate were stained with Warthin-Starry silver stain, and microscopic examination of these stained slides did not reveal any findings indicating Bartonella infection. The pus aspirate was cultured on sheep blood agar, MacConkey agar, chocolate agar, and in thioglycolate broth. The cultured agar plates and the thioglycolate broth were sealed and incubated in $5 \% \mathrm{CO}_{2}$ at $37^{\circ} \mathrm{C}$. We examined the plates daily for 4 weeks for the evidence of microbial growth, but we did not detect $B$. henselae growth. We did not perform serological testing of $B$. henselae antibodies because the kits were unavailable.

DNA was extracted from the aspirate using QIAamp DNA Tissue Mini kit (QUIAGEN, Hilden, Germany). We performed PCR of the purified template DNA using primers described by Jensen et al. [4] and Maggi and Breitschwerdt [5]. The primers reported by Maggi and Breitschwerdt [5] targeted 2 consensus regions of the 16S23S rRNA intergenic spacer region (ITS) of Bartonella species, whereas the primers described by Jensen et al. [4] targeted only 1 region of ITS [4]. Universal 16S rRNA primers were used as the internal control [6]. PCR amplification with the primers described by Maggi and Breitschwerdt [5] yielded amplicons with sizes (459 and 684 bp) corresponding to the 2 consensus regions of the $16 \mathrm{~S}-23 \mathrm{~S}$ rRNA ITS of $B$. henselae. No band was obtained with the universal 16S rRNA primers, and only nonspecific bands were observed with the primers described by Jensen et al. [4]. The PCR products were sequenced using ABI PRISM BigDye Terminator Cycle Sequencing kit (Applied Biosystems, Foster city, CA, USA) and resolved using ABI PRISM 3730XL analyzer (Applied Biosystems), thereby revealing a consistent sequence corresponding to the 16S23S intergenic spacer region of $B$. henselae strain Houston-1 (L35101) with 99.7\% identity. The next closest alignment (93\% identity) of the PCR-amplicon sequence was to the corresponding sequence of B. koehlerae (AF312490).
For the first 3 days, the patient received clarithromycin, which was then switched to azithromycin because of poor compliance. Her condition improved without any complications, and lymphadenopathy was resolved within 2 weeks.

In immunocompetent children and adolescents, CSD is usually self-limiting and does not require any antibiotic treatment. However, azithromycin has shown clinical efficacy [7], as in our case. The atypical symptoms of CSD such as bacillary angiomatosis, bacillary peliosis hepatis, Parinaud's oculoglandular syndrome, encephalopathy, endocarditis, and bacteremia may progress to more serious consequences in 5-20\% of CSD patients [8]. Although some of these symptoms are fatal if left untreated, a treatment protocol for atypical CSD has still not been established because of the lack of epidemiologic, clinical, and diagnostic data [8]. To date, CSD is underdiagnosed in many countries, probably because of the difficulty in isolation with conventional culture [9]. CSD misdiagnosis is another potential risk and may lead to unnecessary treatment [8]. Therefore, clinical recognition and accurate diagnosis of CSD is essential.

The clinical diagnosis of CSD is based on the detection of an enlarged lymph node and possibly a skin lesion at the contact site. Clinicians should investigate the patient's contact history with cats, dogs, rodents, fleas, ticks, or other blood sucking arthropods. The laboratory diagnostic approaches include culture, histological, serological, and molecular methods [10].

The sample is cultured on blood agar containing 5\% sheep or horse blood and $100 \mathrm{mg} / \mathrm{L}$ hemin and incubated in $5 \% \mathrm{CO}_{2}$ at $37^{\circ} \mathrm{C}$. B. henselae grows slowly, and a primary isolate is typically obtained in about 12-14 days. However, the culturing of Bartonella is still a complicated process; therefore, only a small number of CSD cases have been diagnosed using this approach [11]. In our study, we inoculated the sample on blood agar and chocolate agar plates and incubated them for 4 weeks, but no Bartonella growth was detected. In addition to culturing, the aspirate or biopsy specimen may be subjected to histopathologic examination after staining with Warthin-Star- 
ry silver stain, which is neither highly specific nor sensitive for Bartonella species [10].

The serological methods for diagnosis indicate seroconversion or significant increase in the serum anti-B. henselae antibody titer within 15 days. However, the commercialized serological diagnostic kits do not yield consistent results, and the sensitivity of these kits are lesser than those of molecular methods [12].

Since $B$. henselae cannot be detected using culture or histology and serological tests lack sensitivity, a confirmatory diagnosis of CSD is essentially based on PCR [10]. Lymph node aspirate can be easily obtained by fine-needle aspiration, thereby obviating the need for an excision biopsy. Molecular methods have successfully identified the causative organism to the strain-specific level with enhanced sensitivity and specificity [13].

The initial PCR performed using DNA extracted from $0.1 \mathrm{~mL}$ of pus specimen was negative for all the abovedescribed primers. A second PCR performed with DNA extracted from $1 \mathrm{~mL}$ of pus specimen showed bands of the expected sizes with both Maggi and Breitschwerdt primers [5]; the absence of these bands in the first PCR may be attributed to the insufficient amount of template DNA to overcome the PCR inhibitors in the pus specimen [14].

In our study, the internal control (universal 16S rRNA primer) did not show any amplification. To determine the reason for this finding, we aligned the 16S rRNA primer sequence with the nucleotide sequence of $B$. henselae and found that only the forward primer had a matching sequence alignment. Although this primer was designed to target the conserved 16S rRNA sequence common to most bacterial species, it failed to identify the 16S rRNA sequence of $B$. henselae. Moreover, since the 16S rRNA genes of Bartonella spp. show more than 97.8\% similarity [13], the 16S rRNA gene may not be a suitable target for Bartonella species identification.

This is the third reported case of cat scratch disease that was confirmed by PCR for B. henselae in Korea. The 2 previous reports had used the primer sets for the gitA gene described by Margolis et al. [15] and the primer sets for the pap31 gene described by Zeaiter et al. [14], which employed nested PCR. However, in comparison to conventional PCR, nested PCR is more cumbersome, and the samples are prone contamination; thus, this approach may not be widely applicable. Therefore, we used conventional PCR to amplify the extracted template DNA with primers described by Maggi and Breitschwerdt [5], which targetted the 16S-23S rRNA ITS region.

In Korea, few clinical or epidemiological studies on $B$. henselae have been performed; the dearth of studies can be attributed to the absence of established diagnostic methods and the lack of clinical recognition for $B$. henselae infections. CSD can be overlooked or misdiagnosed in the absence of proper molecular diagnostic tools. Therefore, clinicians must consider CSD while diagnosing a patient presenting with lymphadenopathy and/or fever and thoroughly investigate the patient's contact history with cats or dogs. As the incidence of CSD increases, appropriate diagnostic tools that can be widely applied in clinical epidemiology may be established.

\section{REFERENCES}

1. Jackson LA, Perkins BA, Wenger JD. Cat scratch disease in the United States: an analysis of three national databases. Am J Public Health 1993;83:1707-11.

2. Chung JY, Han TH, Kim BN, Yoo YS, Lim SJ. Detection of Bartonella henselae DNA by polymerase chain reaction in a patient with cat scratch disease: a case report. J Korean Med Sci 2005;20:888-91.

3. Chung JY, Koo JW, Kim SW, Yoo YS, Han TH, Lim SJ. A case of cat scratch disease confirmed by polymerase chain reaction for Bartonella henselae DNA. Korean J Pediatr 2005;48:789-92.

4. Jensen WA, Fall MZ, Rooney J, Kordick DL, Breitschwerdt EB. Rapid identification and differentiation of Bartonella species using a single-step PCR assay. J Clin Microbiol 2000;38:1717-22.

5. Maggi RG and Breitschwerdt EB. Potential limitations of the 16S-23S rRNA intergenic region for molecular detection of Bartonella species. J Clin Microbiol 2005;43:1171-6.

6. Loffler FE, Sun Q, Li J, Tiedje JM. 16S rRNA gene-based detection of tetrachloroethene-dechlorinating Desulfuromonas and Dehalococcoides species. Appl Environ Microbiol 2000;66:1369-74. 
7. Bass JW, Freitas BC, Freitas AD, Sisler CL, Chan DS, Vincent JM, et al. Prospective randomized double blind placebo-controlled evaluation of azithromycin for treatment of cat-scratch disease. Pediatr Infect Dis J 1998;17:447-52.

8. Massei F, Gori L, Macchia P, Maggiore G. The expanded spectrum of bartonellosis in children. Infect Dis Clin North Am 2005;19:691711.

9. Ben-Ami R, Ephros M, Avidor B, Katchman E, Varon M, Leibowitz C, et al. Cat-scratch disease in elderly patients. Clin Infect Dis 2005; 41:969-74.

10. Eglantin F, Hamdad F, El Samad Y, Monge AS, Sevestre H, Eb F, et al. The diagnosis of cat-scratch-disease-associated adenitis: diagnostic value of serology and polymerase chain reaction. Pathol Biol (Paris) 2008;56:461-6.

11. La Scola B and Raoult D. Culture of Bartonella quintana and Bartonella henselae from human samples: a 5-year experience (1993 to 1998). J Clin Microbiol 1999;37:1899-905.

12. Bergmans AM, Peeters MF, Schellekens JF, Vos MC, Sabbe LJ, Ossewaarde JM, et al. Pitfalls and fallacies of cat scratch disease serology: evaluation of Bartonella henselae-based indirect fluorescence assay and enzyme-linked immunoassay. J Clin Microbiol 1997;35:1931-7.

13. Fenollar F and Raoult D. Molecular genetic methods for the diagnosis of fastidious microorganisms. APMIS 2004;112:785-807.

14. Zeaiter Z, Fournier PE, Raoult D. Genomic variation of Bartonella henselae strains detected in lymph nodes of patients with cat scratch disease. J Clin Microbiol 2002;40:1023-30.

15. Margolis B, Kuzu I, Herrmann M, Raible MD, Hsi E, Alkan S. Rapid polymerase chain reaction-based confirmation of cat scratch disease and Bartonella henselae infection. Arch Pathol Lab Med 2003;127:70610. 